\title{
Cushing's syndrome Secondary to Bilateral Functioning Adrenocortical Adenomas
}

\author{
Endocrinology Department, Derriford Hospital, Plymouth \\ Zeenat Banu, Haider Khan, Saiful Kassim
}

\section{Case Summary:}

A 67 years old lady was admitted with profound weakness and weight loss for 3-4 months. She has background of primary hyperparathyroidism, hypertension and osteoporosis

Examination revealed BP of $175 / 90 \mathrm{mmHg}$, slightly plethoric with proximal myopathy and bruising. CTTAP revealed bilateral axillary and para aortic lymphadenopathy and right adrenal adenoma $32 \times 26 \mathrm{~mm}$.CT guided biopsy which showed CLL. She was worked up for hypercortisolism.

\begin{tabular}{|c|c|c|c|c|c|c|}
\hline $\begin{array}{l}\text { Table. } 1 \\
\text { Investigations } \\
24 \text { hr urinary free cortisol (1st) }\end{array}$ & $\begin{array}{l}\text { Patients values (Normal } \\
\text { values) } \\
429 \text { (50-300nmol/24hr) }\end{array}$ & Table:2 & Peripheral & $\begin{array}{l}\text { Right } \\
\text { adrenal } \\
\text { vein }\end{array}$ & $\begin{array}{l}\text { Left } \\
\text { adrenal } \\
\text { vein }\end{array}$ & A:U Ratio \\
\hline $24 \mathrm{hr}$ urinary free cortisol $\left(2^{\text {nd }}\right)$ & 306 (50-300nmol/24hr) & $\begin{array}{l}\text { Baseline } \\
\text { Cortisol }\end{array}$ & 475 & 2559 & 740 & $3.45(>2)$ \\
\hline Plasma ACTH (1st) & $6(0.0-40 \mathrm{ng} / \mathrm{l})$ & $\begin{array}{l}\text { Baseline } \\
\text { Aldosterone }\end{array}$ & 146 & 3000 & 999 & \\
\hline $\begin{array}{l}\text { Plasma ACTH }\left(2^{\text {nd }}\right) \\
24 \text { hr urinary Metadrenaline }\end{array}$ & $\begin{array}{l}<5(0.0-40 \mathrm{ng} / \mathrm{l}) \\
0.58(0.0-2.0 \mu \mathrm{mol} / 24 \mathrm{hr})\end{array}$ & $\begin{array}{l}\text { 15min Post } \\
\text { ACTH }\end{array}$ & & & & \\
\hline 24 hr urinary Normetadrenaline & $0.78(0.0-4.3 \mu \mathrm{mol} / 24 \mathrm{hr})$ & Cortisol & 719 & 17500 & 10743 & $1.62(<2)$ \\
\hline Plasma aldosterone & 71 (40-310ng/L) & Aldosterone & 260 & 14540 & 10570 & \\
\hline concentration & & $\begin{array}{l}\text { 25min Post } \\
\text { ACTH }\end{array}$ & & & & \\
\hline Plasma renin activity & 2.8 (3-40mU/L) & Cortisol & 826 & 17500 & 17500 & $1.0(<2)$ \\
\hline $\begin{array}{l}\text { Dehyroepiandrosterone } \\
\text { sulphate }\end{array}$ & $5.8(0.26-6.68 \mu \mathrm{mol} / \mathrm{L})$ & Aldosterone & 285 & 13160 & 14970 & \\
\hline Androstenedione & $21.2(0.0-3.5 \mathrm{nmol} / \mathrm{l})$ & & & & & \\
\hline
\end{tabular}

She had an overnight $1 \mathrm{mg}$ DST which showed unsuppressed 8am cortisol levels of $156 \mathrm{nmol} / \mathrm{L}$. Her $48 \mathrm{hr}$ DST results were $793 \mathrm{nmol} / \mathrm{L}$ to $158 \mathrm{nmol} / \mathrm{L}$. She had an MRI adrenals which revealed right adrenal of $3.3 \mathrm{~cm}$ and left adrenal of $1.4 \mathrm{~cm}$ (Image 1 and 2) with normal MRI pituitary. DEXA Scan showed osteoporosis. Octreotide scan showed no uptake in both adrenals. Hormonal dynamic studies (AVS) revealed bilateral autonomous secretion of cortisol (Table 2). She underwent laparoscopic bilateral adrenalectomies, commenced replacement therapy with hydrocortisone and fludrocortisone postoperatively. Histopathology showed nodular proliferation of predominantly lipid containing cells suggestive of bilateral adrenal cortical adenomata.

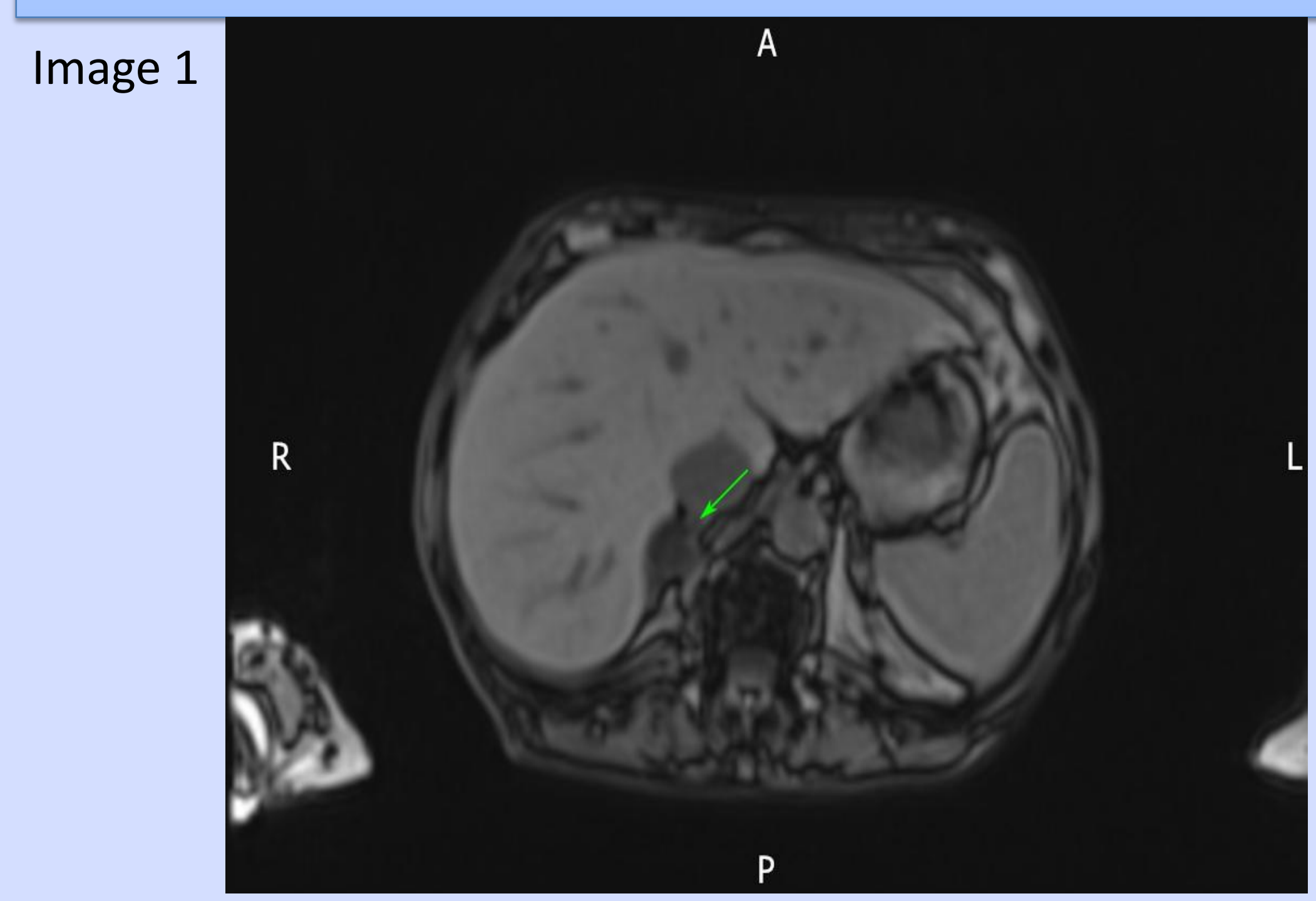

Image 2

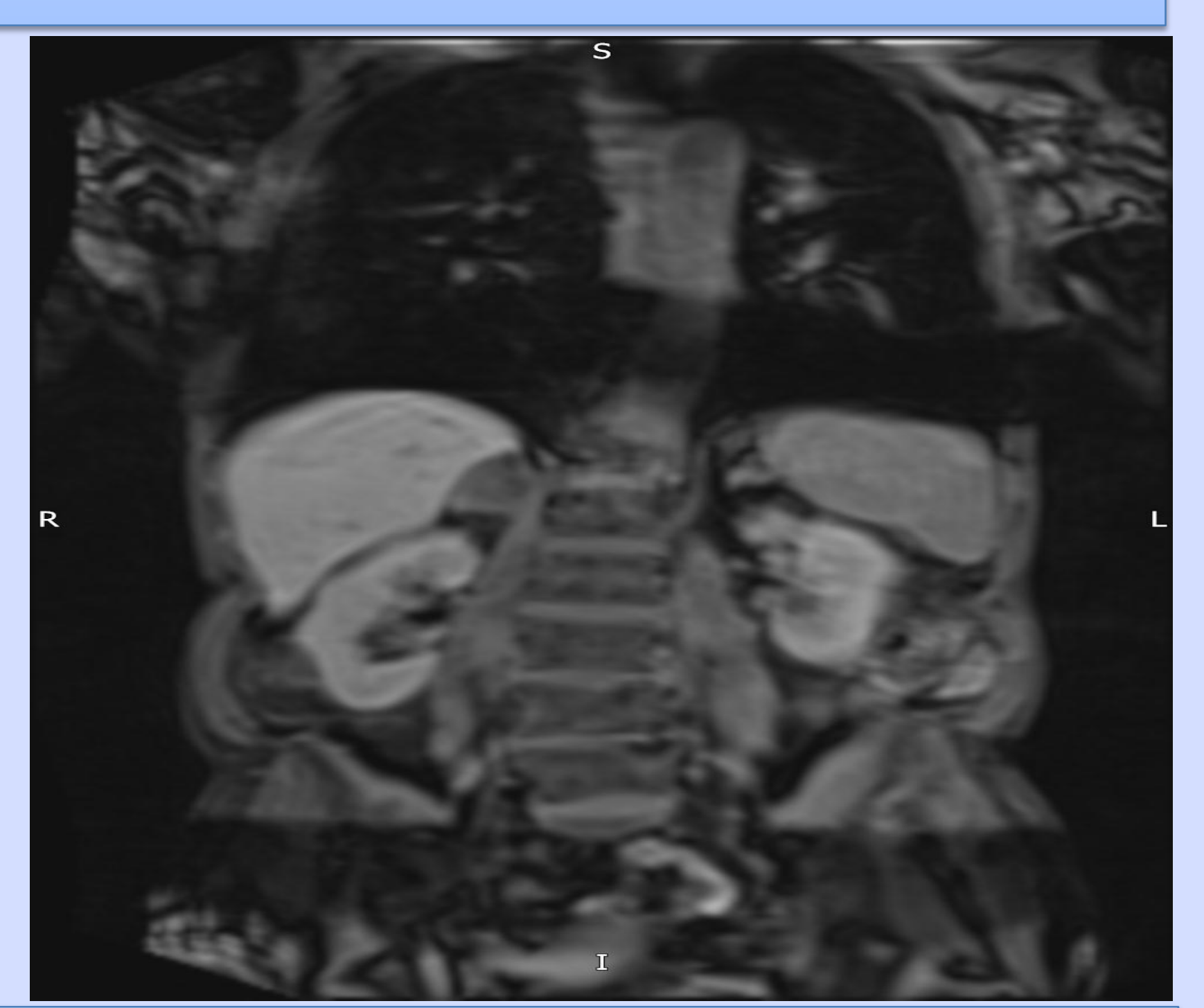

\section{Discussion:}

Bilateral functioning adrenal adenomas are very rare. Only 25 cases are reported since 1977. Abnormal adrenal expression of receptors for various hormones can lead to ACTH independent bilateral macronodular hyperplasia (AIMAH). These are multiple nodules, not encapsulated with hypertrophic adjacent areas. However cortisol producing adrenal adenomas are usually single, unilateral and encapsulated, usually associated with suppressed ACTH levels leading to atrophy of adjacent non-nodular areas.

\section{References:}

1-lino K, Sasano H, Nagura H, et al. Adrenal adenoma with bilateral adrenocortical nodular change in a patient with Cushing's syndrome. Clin Endocrinol (Oxf) 1997; 47:371.

2-van den Berg G, Frölich M, Veldhuis JD, Roelfsema F. Combined amplification of the pulsatile and basal modes of adrenocorticotropin and cortisol secretion in patients with Cushing's disease: evidence for decreased responsiveness of the adrenal glands. J Clin Endocrinol Metab 1995; 80:3750. 